\title{
Management of personality disorders in acute in-patient settings. Part 2: Less-common personality disorders
}

\author{
Leonard Fagin
}

\begin{abstract}
Less-common personality disorders affecting patients admitted to in-patient units are discussed in some detail. These disorders are rarely a direct cause of admission, but they are often associated with other Axis I disorders and can therefore be obstacles to successful treatment. A knowledge of the clinical picture and underlying dynamics and an awareness of countertransference feelings that these patients are likely to provoke can enhance therapeutic alliances and improve the chance of effective care plans.
\end{abstract}

This is the second of two articles that tackle the management problems presented when patients with personality disorders are admitted in crisis to the in-patient unit. The first article (Fagin, 2004, this issue) discusses borderline personality disorder.

In this second article, I discuss clinical management problems associated with patients who present to the acute unit with less-common personality disorders. Such disorders also often complicate a diagnosed Axis I disorder, and successful treatment approaches aimed at dealing with that diagnosis invariably depend on concerted efforts to handle appropriately the personality dimension, which often 'gets in the way'.

The different characteristics of personality configurations make it impossible to state formulaic approaches to problems, and therefore initially it may be prudent to visit currently defined diagnostic categories and then, with the help of descriptive clinical pictures, to try to see whether the in-patient acute environment can contribute to their management. I acknowledge that a number of commentators are critical of these nosological entities, but accept that for the moment they are the best we have (Coid, 2003) and, following most researchers, I have relied on the DSM-IV classification (American Psychiatric Association, 1994) (Box 1). I have merged my discussion on schizoid and schizotypal personality disorders as they tend to be treated similarly in the UK. I have also decided to exclude from this article reference to narcissistic personality disorder, as many commentators believe that it is a diagnosis of questionable validity and the likelihood of a patient with this personality dimension being admitted to hospital is exceedingly small.

\section{Paranoid personality disorder}

In paranoid personality disorder, the key features are ego-syntonic, so it is not uncommon for relatives, friends or work colleagues to lose patience with
Box 1 DSM-IV classification of personality disorders
Cluster A (odd, eccentric types)
- Paranoid personality disorder
- Schizoid personality disorder
- Schizotypal personality disorder
Cluster B (dramatic, emotional or erratic types)
- Borderline personality disorder
- Narcissistic personality disorder
- Antisocial personality disorder
- Hysterical and histrionic personality disorder
Cluster C (anxious and fearful types)
- Obsessive-compulsive personality disorder
- Avoidant personality disorder
- Dependent personality disorder

Leonard Fagin is a consultant psychiatrist and the Clinical Director of the North East London Mental Health NHS Trust. He also holds the post of honorary senior lecturer at University College London (South Forest Centre, 21 Thorne Close, Leytonstone, London E11 4HU, UK). Among his research interests he has studied the psychological effects of unemployment, occupational stress, standards of care in in-patient units and the effects of deinstitutionalisation. 
their constant suspiciousness and accusations. Their hyperawareness of 'what may lie beneath the surface' can make people with the disorder intolerable to those around them, and requires considerable expenditure of energy and time on their behalf when they become patients (Gabbard, 2000).

A diagnosis of paranoid personality disorder implies that the patients' thinking is not strictly delusional, but that their cognitive thinking style involves a distortion of reality, often determined by their past history. This rigidly held outlook is translated into their pattern of relationships, characterised by a constant search to confirm negative suspicions about those around them. Although they adopt the role of victim, they also need to control their persecutors, reflecting by this an unacknowledged low self-esteem, often compensated by grandiosity. This makes them vulnerable to the fear of being humiliated by people in authority, which is one of the major obstacles to a therapeutic relationship. Their verbal attacks on staff often provoke defensive explanations, which are received with even more suspicion.

Most patients with paranoid personalities are not known to psychiatric services, and those who are may initially have been referred to psychiatric out-patient clinics by their families or employer. Very occasionally, however, they are admitted to hospital as a result of an aggressive physical attack on a close relative or a colleague at work, incited by their suspicions. Initial assessment is likely to involve a differential diagnosis of paranoid schizophrenia, but once this alternative has been discarded, the challenge is to see how admission, even if brief, can be used as a platform for further psychiatric or psychological interventions. More often than not, such admissions end up confirming the patient's paranoid suspicions about services, resulting in a wasted opportunity. Their response will greatly depend on the ability of staff to contain their paranoid projections without counterattacking or responding in a defensive manner. Staff will need to withstand the onslaught of accusations and demeaning remarks. Any success in this regard has the possible corollary of establishing a bridge of contact that can be crossed at some time in the future. One way of handling this is to acknowledge the efforts patients have to expend to keep those around them at a safe emotional distance. This approach may eventually allow the patients to acknowledge their own fears and weaknesses, something that they desperately defend themselves against. This shift, which will happen in the patients' own time and in an enabling therapeutic framework, will permit patients to contemplate an alternative view to their previously rigid and stereotypical perception of the world around them.

I have already mentioned a propensity to violence as one of the factors likely to bring paranoid individuals into hospital. This risk will remain present during the admission and require strategies to prevent it (Box 2).

\section{Box 2 Violence-prevention strategies (adapted from Gabbard, 2000)}

Help patients to save face

Avoid arousing further suspicion

Be prepared to be openly firm when necessary

Help patients to feel that they are in control

Encourage verbalisation rather than physical acting out

Give plenty of breathing space

Tune in to own countertransference denial of violence
Staff must be particularly aware of patients' low self-esteem and of their need to keep this hidden from themselves as well as from the outside world

Staff should explain each and every movement that they make and should avoid ingratiating friendliness

This may involve straight talking; when decisions have to be made that could be perceived as persecutory, staff should clearly explain how they have come about and in whose interests they would be

Staff must openly acknowledge why patients have construed the world around them as they have and must respect their need for autonomy

Staff should sense and support patients' experience of anger and, together with them, consider the consequences of violence

Avoid close seating arrangements and physical contact

Remember that women are as likely as men to assault staff (Tardiff et al, 1997). Be aware of your own aggressive and provocative feelings 


\section{Schizoid and schizotypal personality disorders}

'If a man does not keep pace with his companions, perhaps it is because he hears a different drummer. Let him step to the music he hears, however measured and far away.'

(Thoreau, 1937; 1950 reprint: p. 290)

Although there is still discussion about the inclusion of these diagnoses among the Axis II disorders, the persistence of social detachment and blunting of affect, magical thinking not amounting to delusional processes, and eccentricity of behaviour have placed this nosological entity within the disorders of personality rather than the psychoticspectrum Axis I disorders.

By their very nature, these patients rarely come to the attention of general psychiatrists and even more rarely to that of in-patient units, unless their social isolation, self-neglect and eccentric, bizarre behaviour lead to their referral under the mistaken diagnosis of schizophrenia. Occasionally, relatives bring them along to a psychiatrist, concerned that they are not 'engaging enough with life'. Some patients share the notion that they are mere shells of personality, with no life or feelings, not sure of who or what they are and, as a result, incapable of establishing or sustaining meaningful emotional relationships. Closeness carries with it the threat of being engulfed or taken over by other personalities, and therefore extinguished, and yet there is a need to remain attached in order to survive: what Guntrip (1968) calls the 'schizoid compromise'. Paradoxically, therefore, not being connected permits people with schizoid personalities to believe that they are defending their 'true self' against being overpowered by others and transformed through these interactions into a 'false self' (Winnicott, 1965: pp. 179-192).

In-patients with schizoid personality features present various management problems (Box 3). On admission, they characteristically seek refuge in their bedrooms or a quiet corner of the unit, and avoid all interactions with staff or other patients, at least until they feel relatively secure. Any group activities are experienced as threats, and attempts at forcing them to participate will be fiercely resisted. As the environment becomes more familiar and less threatening, and in particular if they are able to attach themselves to a member of staff or another patient, with encourgament they may gradually 'thaw out', and this can provide, perhaps for the first time in their lives, an opportunity to explore relatedness in a safe context. The challenge for staff is not to collude with their aloofness and distancing techniques, not to allow them become 'part of the furniture'. Staff must be aware that, although silence can be a form of resistance, it is also communication
Box 3 Interactive issues in the management of patients with schizoid personality disorders

- Accept silence as a powerful form of communication

- Be prepared to feel rejected and distanced

- Be careful of persistent probing

- Accept the patient's rhythm and pace of change

- Validation by other patients often carries more weight than validation by staff

and an attempt at relating, but sharing it in a nonconstrictive way demands extraordinary fortitude and resilience. In a sense, it is a way of accepting the 'true self' that the patient is so desperately trying to defend. Those attempting to relate to such patients will have to examine carefully their own reactions, and perceive what sort of feelings are being engendered in them, as these may give an indication of what the patient is going through or attempting to communicate. The task then is to see how these feelings can be shared with the patient in a way that can be accepted and learnt from.

The possibility of beginning to establish a modicum of relatedness will obviously depend on the degree and severity of withdrawal. Staff may need to be prepared to accept the unfathomableness of some patients and to function as a supportive alter ego in practical areas such as personal care, reality testing and basic interpersonal skills. As they become more comfortable, these patients will begin to accept group activities, an ideal setting in which socialisation and relatedness to peers can be explored. Therapists in these settings will have to bear in mind the possibility that other staff may gang up on individual patients in order to extract information from them or, conversely, simply ignore them and treat them as if they were not there.

The use of antipsychotics in schizotypal personality disorder has also been postulated as an aid to engagement and reduction of withdrawal behaviour (Davison, 2002).

\section{Antisocial personality disorder}

The most challenging of patients are those with antisocial personality disorder, and many are considered untreatable, requiring corrective rather than therapeutic interventions. The disorder is sometimes referred to as psychopathy, sociopathy or character disorder, but these terms have fallen out of favour because of their pejorative connotations. There is still some diagnostic controversy about the term. However, Gabbard (2000) suggests that 
antisocial personality disorder lies on a continuum with narcissistic personality disorder, and that treatability will depend on a dynamic understanding of where each patient is on this continuum. According to the National Institute for Mental Health in England (2003) the epidemiology of this condition indicates a $2-3 \%$ lifetime prevalence, highly weighted in impoverished areas, and the condition is associated with criminality and drug misuse. There is a strong male preponderance, between 4:1 and $8: 1$, but there is a tendency to underdiagnose this disorder in women because of stereotypes. Obstetric complications and perinatal brain injury, lowered autonomic nervous system responsiveness, maternal rejection, physical abuse, negative family environments, and conduct and attention-deficit hyperactivity disorders have all been implicated in the aetiology, but not in a definitive sense.

From a psychodynamic point of view, the absence of superego development and of any attempt at moral justification of aggressive antisocial behaviour or lying in order to avoid responsibility makes these patients poor candidates to respond to any therapeutic approaches, let alone to engender any empathic responses from staff. There are, however, gradations in this area, and some patients are able to experience a degree of guilt or concern, even in the light of repeated antisocial acts, which may make them amenable to interventions.

These interventions are best carried out in residential or in-patient psychotherapy units that work within clearly defined boundaries. An admission to a general psychiatric unit is inadvisable, because the likelihood is that these patients will disrupt the treatment of others by flouting conventions and undermining therapeutic activities and the ability of staff. Some patients charm staff into thinking that they are benefiting from their care, whereas they are going through the motions without any real change; some convince staff that they are victims of circumstances or only 'bad' when they use drugs or alcohol. These patients may take advantage of the kindness or need of staff to be helpful, encouraging them to play down the patient's ruthlessness, even in the light of previous negative experience. Needless to say, they arouse extreme negative responses in staff. To some degree this depends on the setting: countertransference in a hospital is different from that in a correctional institution. Antisocial personalities tend to elicit in staff very polarised feelings: from the desire to punish and seek retribution to the illusion that staff can defeat evil by their kindness and endeavour; from outrage to admiration, hopelessness to fear (Lion, 1999). Added to this are the dangers of being deceived, as these patients regularly distort facts and lie, thereby disabling any semblance of a trusting therapeutic alliance.
Box 4 Predictors of response to therapeutic approach in antisocial personality disorder (after Gabbard \& Coyne, 1987)

Predictors of positive response

- Presence of anxiety

- Axis I diagnosis of depression

- Axis I diagnosis of psychosis other than depression or organic condition

Predictors of negative response

- History of arrest for a felony

- History of repeated lying, use of aliases, conning

- Unresolved legal situation on admission

- History of conviction for a felony

- Hospitalisation as an alternative to imprisonment

- History of violence to others

- Diagnosis on Axis I of organic brain impairment

Patients admitted in crisis should be assessed rapidly and the decision taken either not to treat but to take risk-management actions or, if there is scope for intervention, to refer to the relevant service as soon as possible. While this is happening it is important that strict boundaries and conditions are set regarding aggression, sexual acting out, theft and drug importation, with consequences if these are transgressed. Staff countertransference feelings of disbelief, rationalisation and collusion must be carefully monitored. Fear of assault may lead to loosening of agreed boundaries for fear of provoking a reaction. Any breaking of rules needs to be confronted immediately, so that patients are aware of the consequences of their actions, and they should be encouraged to think and talk before taking impulsive action. Unfortunately, this is not always feasible.

Gabbard \& Coyne (1987) have produced a list of predictors (Box 4) that can be used in in-patient units to assess potential treatability, and they advise that staff should not to be led by 'gut-feeling responses'.

Lion's (1999) advice for dealing with antisocial personality disorder is summarised in Box 5 .

\section{Hysterical and histrionic personality disorders}

Hysterical and histrionic conditions are not properly distinguished in DSM-IV. Hysterical conversion disorder is subsumed under conversion or somatoform disorder and is not related to hysterical personality disorder.

Gabbard (2000) describes both hysterical personality disorder and histrionic personality disorder. 
Box 5 Dealing with patients with antisocial personality disorder (after Lion, 1999)

- Remain sceptical, particularly during assessments

- Do not deny or normalise dangerousness: a charismatic patient can lure staff into a forgiving or permissive response to unacceptable behaviour

- Be aware of polarities in feeling: a patient's assertiveness or even violence can arouse secret awe and admiration one moment, but disgust and punishment the next

- Be aware of the risk of sexual seduction by patients

- Ensure that less experienced staff are supervised by a more experienced clinician

He attributes to them shared behavioural characteristics such as a tendency to labile and shallow emotionality, attention-seeking, disturbed sexual functioning, dependency and helplessness, and selfdramatisation. However, he differentiates hysterical personality as being healthier, whereas histrionic personality is more florid in every way, less subtle and more impulsive, functioning at a much more primitive level. This differentiation is often reflected in individuals' respective success or failure to maintain relationships and work commitments, and in differences of degree of erotic transference wishes.

Although traditionally seen as female disorders, hysterical and histrionic personality disorders have also been extensively documented in men. These have fallen into two broad subtypes: the hypermasculine Don Juan, unable to commit himself to any relationship, and the passive effeminate man, homosexual or heterosexual, usually impotent. In both genders the cognitive style is impressionistic, unable to elaborate detail about the people or world around them, indicating a defensive emotional detachment (la belle indifference), although, paradoxically, they may present with shallow emotionality.

Women with histrionic personality disorder tend to have a history of maternal rejection, which draws them to their fathers for dependency needs. They become 'daddy's little girl' and repress their own sexual maturation and identity.

Women with hysterical personality disorder have usually had more satisfying early relationships with their mothers, but develop intense feelings of rivalry and compete for their fathers' attention. They are more likely to have a history of actual incest. In adulthood they appear to be unaware of their attempts at seductiveness. As a result their own sexuality and experience of intimacy are disturbed and unsatisfactory, as is their choice of partners. It is usually a relationship crisis, leading to dramatic acts of impulsive self-harm, that results in admission to an acute hospital setting by way of the accident and emergency department.

The story is similar for men. In men with histrionic personalities, maternal (and paternal) unavailability may lead them to emulate their mothers, adopting a passive, effeminate role, or their fathers, mimicking hypermasculine cultural stereotypes of masculinity. In those with hysterical personalities, feelings of sexual inadequacy keep men attached to their mothers, again either adopting effeminate or celibate lifestyles, or cause them to overcompensate by shallow efforts at becoming tough 'real men'.

Both men and women with these disorders pose difficulties in in-patient settings, as they often engage in rivalrous relationships with other patients and erotic transferences to staff. They usually see themselves as special, tend to take over groups, where they need to be at the centre of all discussions, and take on other people's problems as part of their own, in a self-referential manner. If thwarted in their attempts, they are likely to become increasingly dramatic, and sometimes become involved in risktaking behaviour in order to attract attention. This often provokes negative countertransference feelings in staff, who then try to ignore their demands, which only reinforces the cycle.

Although long-term work with these patients is the province of the out-patient clinic or psychotherapy department, two principles of patient management can be applied in the in-patient unit that might help patients take on more long-term work.

First, the initial assessment should be used as an opportunity to challenge the patient's cognitive style. However, this assumes that doctors and nurses intuitively know what is happening with these patients. History-taking (which can be a frustrating experience) is therefore extremely important, as it allows patients to describe, perhaps for the first time, their internal world, feelings and expectations.

Second, erotic transference must be effectively managed. Eroticised feelings towards staff can sometimes be very insistent and pervasive, and in an in-patient unit nursing staff are particularly vulnerable to overt or covert advances. Many careers have been blighted because of inappropriate crossing of sexual boundaries, which can also be devastating for patients.

The management of transference involves a close examination of countertransference feelings. There are large hurdles to overcome if this is to be dealt with appropriately, as there seems to be no tradition in the UK for openly discussing sexualised feelings, which are often ignored or denied in patients and staff. Nurses in particular have no vehicle for 
expressing their concern and no support system to rely on, and any suggested breaking of boundaries is immediately responded to in a punitive manner, by suspension or dismissal. Sometimes staff are so frightened of these consequences that they respond to patients' advances with aggression or aloofness, which the patients read as evidence that sexual desires are dangerous or dirty. Another common response by staff is to tell the patient that their feelings are not real, when to the patient they are extraordinarily real. A more appropriate response is to communicate to the patient that sexual or loving feelings do occur but cannot be reciprocated. This acknowledges the reality of the feelings, but places them within a therapeutic process that can help staff to understand some of the patient's inner thoughts and feelings, even if at times it is embarrassing or painful for the patient not to have their desires fulfilled.

\section{Obsessive-compulsive personality disorder}

As opposed to obsessive-compulsive disorder, this personality trait is ego-syntonic, and therefore does not cause distress to patients themselves, but is more likely to affect those with whom they live or work. In many cases, the symptoms are adaptive and can be an aid to successful careers. Excessive attention to detail, preoccupation with morality, values, order, rules and regulations, a tendency to perfectionism that can interfere with task completion, extreme devotion to work at the expense of other pursuits and relationships, as well as parsimoniousness are some of the most common features. The cognitive style of people with obsessive-compulsive personality disorder features rigid control over their emotions and an excessive reliance on logical, narrow-minded thinking.

People with the disorder are not usually referred to psychiatric services and are unlikely to end up in in-patient facilities, unless their defence systems decompensate with marked symptoms of anxiety, depression or self-neglect, usually around middle age. A feature of their presentations is the need to become perfect patients, attempting to control their anger, sadness or despair. This sometimes takes the form of rambling, circumstantial conversations that stray from the matter in hand and provoke staff to want to disengage or to prompt patients into action.

If an individual with the disorder is admitted, staff should focus on the patient's feelings rather than facts or words. Groups can be very effective in challenging a patient's attempts to hide behind a smokescreen. Initially, however, it might be necessary to respect the patient's defences. Countertransferential feelings can be challenged and dismantled when the patient feels safe enough to disclose the profound feelings of insecurity and lowered self-esteem that are the kernel of this personality configuration.

\section{Avoidant personality disorder}

Although linked by some researchers with schizoid personality disorder, avoidant personality disorder differs in that those affected show a need for close relationships, hampered by powerful fears of rejection and failure. They have an intense dread of criticism and dismissal in both public and private encounters. There may be strong overlap with social phobias.

It is unlikely that such patients will be admitted to hospital unless they present with another, concomitant Axis I diagnosis. At the heart of these presentations is shame and self-exposure of internally perceived inadequacies.

Dynamic and cognitive-behavioural approaches are the treatment of choice in out-patient environments, but the following clinical strategies are useful for patients admitted to hospital:

- offer an empathic response to their embarrassment, coupled with encouragement to face the feared situation;

- consider prescription of a selective serotonin reuptake inhibitor;

- use psychoeducational approaches to combat anxiety.

\section{Dependent personality disorder}

Dependent personality disorder also regularly presents as a comorbid condition with another Axis I disorder such as depression, bipolar disorder, alcohol dependence, anxiety or eating disorder. Intense submissiveness and clinginess, linked with difficulties in decision-making and a tendency to relinquish responsibility to others, make it an important personality trait that affects the management of the Axis I disorder. Of note here is that the person targeted as the caretaker can experience these demands as hostile and aggressive. These responses are sometimes experienced in the countertransference as well.

Patients with the disorder are likely to become attached to staff on the unit or in the out-patient department, and they fear disastrous consequences if they are discharged from hospital care. Improvement is therefore seen as a potential threat.

Bearing this in mind, clinical strategies should focus on the patients' responsibilities and independence. Staff must feel comfortable in frustrating 
patients' dependency needs, making them take decisions and promoting independent thinking and action. It is important to discuss with these patients their fear of becoming better, and thus separate and independent: time-limitations might help them to accept the fact that their symbiotic relationship will have to end. Staff must be attuned to countertransference feelings of contempt and the urge to bask in idealised roles attributed to them by patients.

\section{References}

American Psychiatric Association (1994) Diagnostic and Statistical Manual of Mental Disorders (4th edn) (DSM-IV). Washington, DC: APA.

Coid, J. (2003) Epidemiology, public health and the problem of personality disorder. British Journal of Psychiatry, 182 (suppl. 44), s3-s10.

Davison, S. E. (2002) Principles of managing patients with personality disorder. Advances in Psychiatric Treatment, 8 $1-9$.

Fagin, 1. (2002) Management of personality disorders in acute in-patient settings. Part 1: Borderline personality disorders. Advances in Psychiatric Treatment, 10, 93-99.

Gabbard, G. O. (2000) Psychodynamic Psychiatry in Clinical Practice. Washington, DC: American Psychiatric Press.

Gabbard, G. O. \& Coyne, L. (1987) Predictors of response of antisocial patients to hospital treatment. Hospital and Community Psychiatry, 38, 1181-1185.

Guntrip, H. (1968) Schizoid Phenomena, Object Relations and the Self. New York: International Universities Press.

Lion, J. (1999) Countertransference in the treatment of the antisocial patient. In Countertransference Issues in Psychiatric Treatment (ed. G. O. Gabbard). Washington, DC: American Psychiatric Press.

National Institute for Mental Health in England (2003) Personality Disorder: No Longer a Diagnosis of Exclusion. Policy Implementation Guidance for the Development of Services for People with Personality Disorder. London: Department of Health. http://www.nimhe.org.uk/downloads/ PDFinal.pdf

Tardiff, K., Marzuk, P. M., Leon, A. C., et al (1997) Violence by patients admitted to a private psychiatric hospital. American Journal of Psychiatry, 154, 88-93.

Thoreau, H. D. (1937) Walden and Other Writings. New York: Random House.

Winnicott, D. W. (1965) The Maturational Processes and the Facilitating Environment: Studies in the Theory of Emotional Development. New York: International Universities Press.

\section{Multiple choice questions}

1 Patients with paranoid personality disorder:

a regularly present with crises that lead to in-patient admission

b should have a risk assessment carried out if admitted

c will need to be confronted about their paranoid suspicions

d will require constant nursing observation

e should be approached only by selected staff who are not involved in their paranoid suspicions.

2 Patients with a diagnosis of antisocial personality disorder:

a should be barred from admission to an in-patient unit if there is a current court case against them

b have a worse prognosis if they experience anxiety and depression c will need to be informed that the admission is only to assess their treatability

d should always be disbelieved

e if female, should preferably have a female as their primary nurse.

3 Patients with schizoid personality disorder:

a are likely to pose a physical threat to staff

b will prefer one-to-one interactions with other patients or staff

c will require direct confrontation in order to break self-imposed isolation

$\mathrm{d}$ are impervious to engagement with occupational therapeutic activities

e will not make demands on staff for attention.

4 As regards patients with histrionic personality disorder:

a female patients will assume that staff are aware of their needs without the need to communicate them

b male patients are invariably homosexuals

c erotic feelings towards staff will need to be ignored

d patients who seek attention are likely to threaten staff with risk-taking behaviour

e these individuals often present with physical complaints.

5 State whether the following statements are true or false:

a patients with obsessive-compulsive disorder are likely to be admitted when their rituals fail to reduce levels of anxiety

b patients with obsessive-compulsive disorder respond well if in-patient staff prevent them from carrying out their rituals

c a patient with a dependent personality disorder will benefit from staff attempts to promote independent decision-making

d negative feelings in staff towards patients with personality disorders are not acceptable and are clinically dangerous

e patients with avoidant personality disorder are similar to those presenting with schizoid features in that both avoid close relationships.

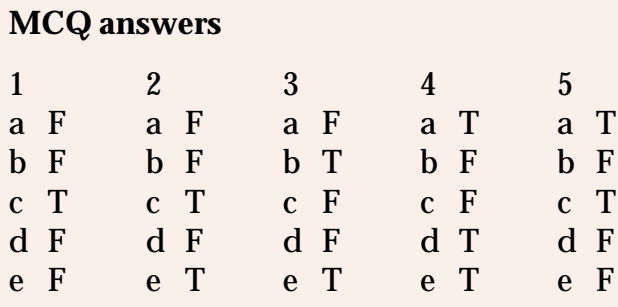

